\title{
Dynamical Evolution in Hickson Compact Groups using Intragroup Light
}

\author{
C. Da Rocha ${ }^{1,2}$, B. L. Ziegler ${ }^{1}$, and C. Mendes de Oliveira ${ }^{3}$ \\ ${ }^{1}$ Institut für Astrophysik Goettingen, Germany ${ }^{2}$ DAS/INPE, Brazil ${ }^{3}$ IAG/USP, Brazil \\ email: rocha@astro.physik.uni-goettingen.de
}

\begin{abstract}
Most of the galaxies in the local universe are located in groups, in particular in small groups, and most of the transformations suffered by galaxies located in today's clusters are likely to have occurred in groups at higher redshifts. Understanding the formation and evolution of groups is essential to understand the whole picture of structures and galaxy build-up. Using multi-band photometry we studied the intragroup light component (IGL) observed in compact groups of galaxies in a subsample of Hickson's catalogue, an efficient tool for determining its stage of dynamical evolution and for mapping its gravitational potential. Applying the OV_WAV package, a wavelet based technique, we can identify the IGL independently of the main contaminating effects. The fractions of IGL detected range from $11 \%$ to $46 \%$, with one group with no IGL detected. The colors are consistent with those for old stellar populations and the mean surface brightnesses range from 24.8 to $28.4 \mathrm{~B}$ mag $\operatorname{arcsec}^{-2}$. Using the IGL, along with other dynamical evolution indicators, we could establish an evolutionary sequence for our sample.
\end{abstract}

Keywords. galaxies: evolution-galaxies: interactions - intergalactic medium-dark matter
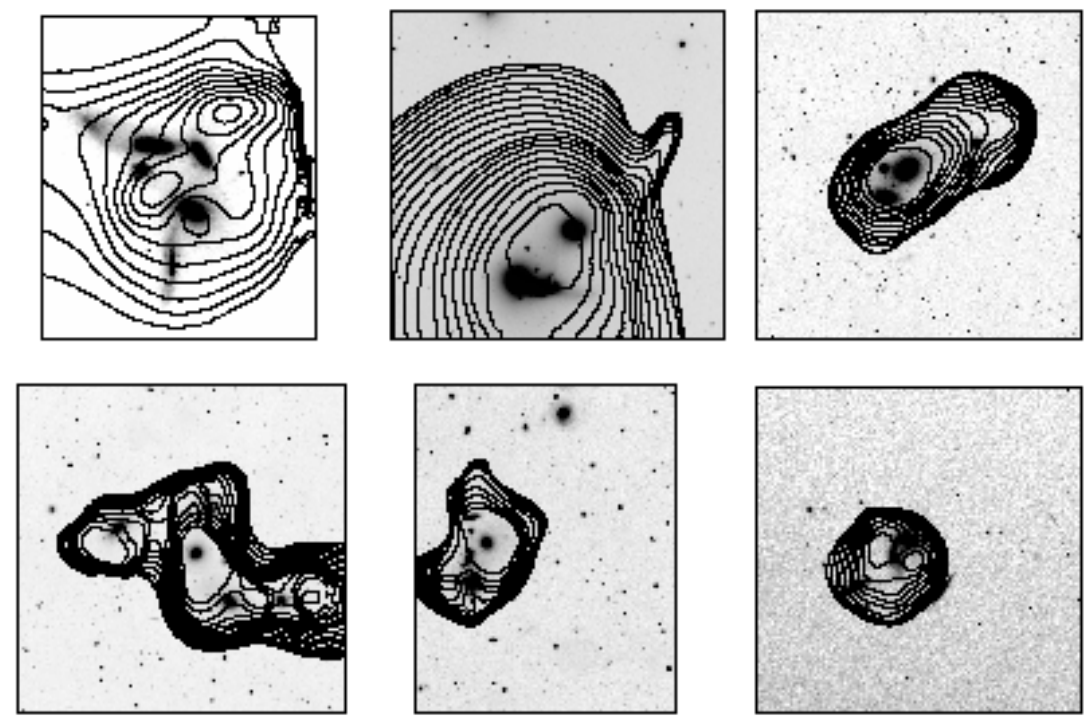

Figure 1. $B$ band images of six of the seven HCGs analyzed in our sample with the IGL overplotted as contour curves. From left to right, top to bottom. HCG 79 and 90 in advanced stages of dynamical evolution, with large IGL fractions (35 to 45\%) and advanced galaxy interaction stages; HCG 51, 15, 35 and 95 in an intermediary stage, with IGL detection with irregular shapes (fractions between 11 to $26 \%$ ), some morphological distortions, on-going interaction and HI deficiency; and HCG 88 (not displayed) in an initial stage of evolution with no IGL detected, no stripped HI and a few signs of interaction. 